tracing taken as above described while the cylinder was revolving slowly. The patient was a young woman, A. C., suffering from well-marked symptoms of Graves's disease in an early stage. The tremor could be distinctly felt when her hand was loosely placed in the hand of the observer. The tracing was taken with her right hand resting lightly on the tambour. The movements occur at the rate of 11 per second, and their amplitude is slight, though varying within small limits. Numerous tracings were taken from this patient, and also from another young woman, B. A., who also presented well-marked symptoms of Graves's disease in the early stage. All the tracings had the same characters, and the rate was practically identical in both patients, the maximum of numerous countings being 11.5 per second in A. C., and the minimum 10.8 per second in E. A. A study of the extended series of tracings from these two cases appears to show that even this slight difference is due to imperfections in the apparatus, and that, within the limits of experimental errat, the rate in these cases was almost identical. Fig. 4 is a reproduc-

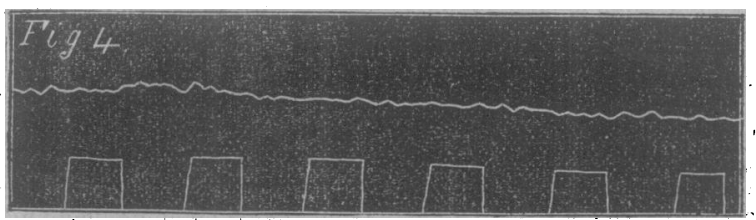

tion of a tracing taken with a more rapidly revolving cylinder. Though the general aspect is, for this reason, different, the rate at which the movements occur (11.3 per second) is almost the same as in Fig. 3, and the two tracings have essentially the same characters.

The next tracing (Fig. 5) is from a man, W. H. H., aged 78 ,

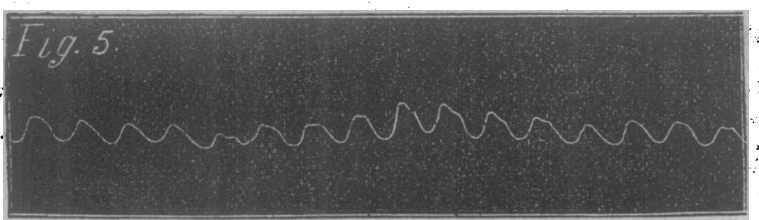

who presented well marked symptoms of paralysis agitans; he was under the care of Dr. Scanes Spicer in the Fulham Infirmary, to whose kindness we are indebted for the opportunity of making this as well as the observations recorded below. Though the tremor was well marked, the patient was still able to do a little light work. The tracing gives a rate of 5.2 per second, but inspection shows that many of the curves give indications of secondary waves. This is beautifully marked in the tracing (Fig. 6) from another case, G. E., an old man, also in the Fulham

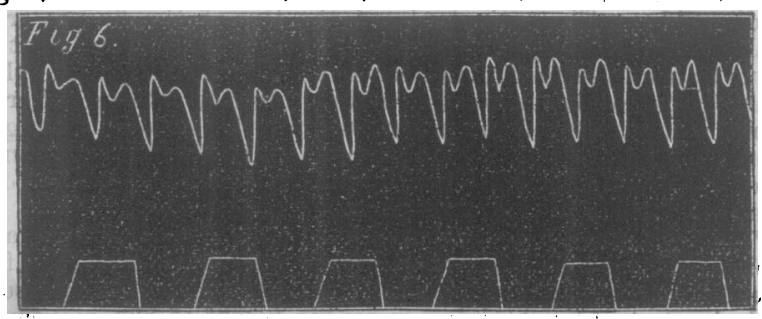

Inflamary, with incapacitating paralysis agitans. Each wave is noted the summit with singular regularity, and is clearly compoyindad of two waves. The larger waves give a rate of 5.1

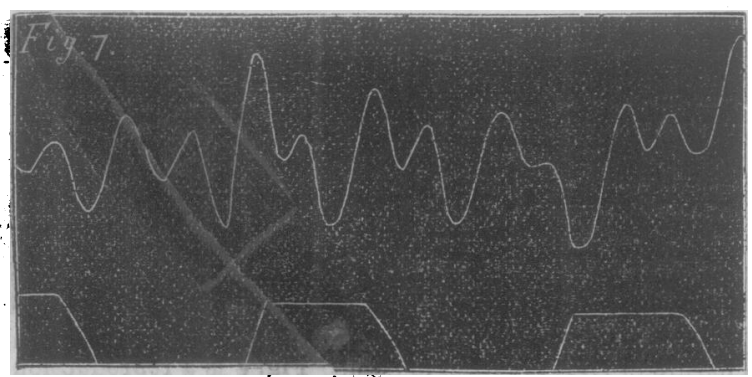

per second ; and, as each is double, the true rate is 10.2 per second, which is about the average rate of the oscillations in health, the difference lying in an alteration in the amplitude, and in the fusing of each alternate wave with the one immediately preceding. This point is further elucidated in another tracing (Fig. 7), taken from this case on a more rapidly revolving drum. The lever, as it descends, is caught by the secondary wave, and again thrown up, very much as in a tracing of a "dicrotic" pulse. The tremor of paralysis agitans, which ordinarily gives such a tracing as that reproduced in Fig. 5, is thus seen to be due to a fusion of the waves in pairs as above stated.

Tracings were taken from two cases of disseminated sclerosis. In one, A. D., a woman aged 77, a very uniform tracing, reproduced in Fig. 8 , was obtained. The rate is 5.8 per second. A tracing

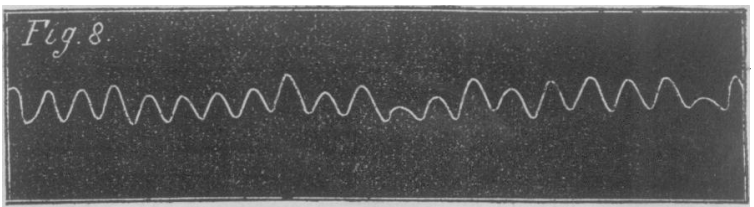

obtained from another case of the same disease in a woman, A.IT., affords evidence of secondary waves, which, viewed in the light afforded by the tracings from the cases of paralysis agitans (Figs. 5, 6, 7), points clearly to a process of fusion. The same effect is extremely well shown in a tracing (Fig. 9) from a case of

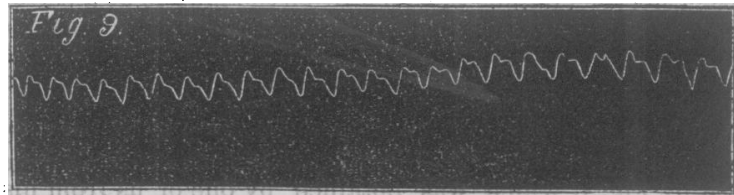

lateral sclerosis with tremor of the arms in a woman aged 63 The rate, if the main oscillations only are counted, is 5.2 ; and, as each oscillation is distinctly double, the true rate is in this cast also 10.4. The next tracing (Fig. 10) is from an old woman aged

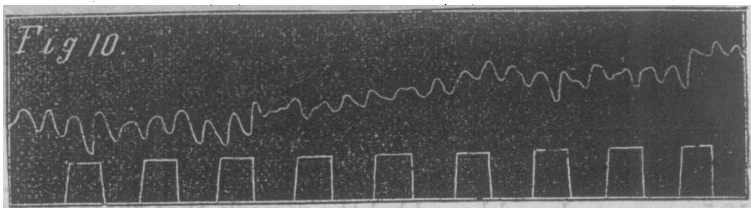

91, who had well-marked senile tremor. The arerage of the oscillations is 5.5 per second. Many of the curves, however, are distinctly notched, so that, as in other instances, we here have, in all probability, an effect of regular summation in pairs.

It would be easy to multiply tracings all giving evidence of $t$ I:e same nature, but enough has been advanced to make it clear that the rate of the tremulous movements in these various forms of nervous disorder does not overpass the normal limits observed by Schäfer and Horsley. The general result of our observations is to show that the special characteristics which clinically distinguish the various forms of tremor are to be traced to two causes: in the first place an exaggeration of the amplitude of the normal vibration, and in the second place to the fusion of vibrations, which has the effect of producing a relatively slow tremor, generally of a rate corresponding to half that of the normal vibration.

\section{A NOTE ON}

FILARIA SANGUINIS HOMINIS:

With a Description of a Male Specimen.

BY. ALFRED GIBBS BOURNE, D.Sc.,

Professor of Biology in the Presidency College, Madras.

Oon knowledge of the adult form of this worm is still incomplete. It was discovered by Dr. Bancroft, in Brisbane, in 1876. It has also been found by Dr. Timothy Richards Lewis, in Calcutta ; Dr. Patrick Manson, in Amoy; Dr. Silva Aranjo and Dr. F. dos Santos, in Brazil. Dr. Bancroft's speciments were described by Dr. Spener Cobbold: Drs. Lewis and Manson have both given descriptions of their spectomens. "Atl these -specimens were imperfect, and are 
stated by Cobbold to have all been females. This authority states in his book (Parasites: a Treatise on the Entoroa of Men and Animals, 1879) that the male parasite is unknown. This was not, however, strictly correct, as Lewis in the paper (Quarterly Journal of Microscopical Science, 1879) referred to by Cobbold, states that he found portions of two specimens: the one was about one inch and a half long, and belonged to a female from which the caudal extremity had been severed, while the other was a fragment of a male worm. Of this latter he says: "It measured half an inch in length, and $t$ of an inch transversely; it was thinner than the female, but of considerably firmer texture-so firm, indeed, that whilst endeavouring to make out its anatomy a considerable portion of it was lost by one of the needles used for dissecting snapping, and carrying a portion of the worm along with it. On tearing the helminth across, the severed surface does not present a ragged edge, but an even outline. The male manifested also a great tendency to coil, and it was only with difficulty that it could be separated from the specimen of the female parasite, around a portion of which it had twisted itself. It is unfortunate that its caudal end, especially, could not be found, as the definite decision of the genus to which it should be referred depends in a great measure on the characters which the posterior end of the male worm presents."

Brigade-Surgeon Sibthorpe has had sereral cases of filarial disease in the General Hospital at Madras, and has always made a search for the adult worm; this has hitherto proved fruitless. Recently, however, after an amputation of a lymphoid scrotum, he found two worms which he had mounted and identified as adult filariæ. He had found embryo filariæ swarming in the patient's blood on the previous erening. He very kindly sent these specimens to me for examination, and I found that one was a female, while the other was a male. The female specimen agrees very closely with Cobbold's figure, but the vagina is everted or protruded. Dr. Smyth, resident surgeon to the hospital, who mounted the specimens, tells me that the eversion took place during mounting; it is probably a normal act during life. The caudal portion is wanting. The male specimen is about an inch and a quarter long; the anterior extremity is wanting, but the caudal extremity is intact, and presents two spicules. The structure of these spicules will doubtless form a valuable specific character. Unfortunately, only one of these spicules remains in situ; the other has dropped out in the mounting and lies nearly isolated on the slide The spicule is broad at its proximal extremity, and gradually tapers until it becomes capillary in character. About half-way down there is a lateral prominence, and when in situ the spicule is folded on itself so that this prominence forms the actual free extremity of the spicule, while the broad end and the capillary end lie near to one another.

A description and figures of this specimen, which, with the exception of Lewis's fragment, is, I believe, the first recorded specimen of the adult male filaria sanguinis hominis, will be published in an ensuing number of the Transactions of the South Indian Branch of the British Medical Association.

It is interesting to note that in this case, as in Lewis's case, the male and female were found in close contiguity:

\section{EMPYEMA FOLLOWING PNEUMONIA.}

BY DONALD W. C. HOOD, M.D.Cantab.,

Senior Physician North-West London Hospital, and Physician West London Hospital.

I wrar to call attention to those cases in which an inflammatory attack connected with the chest has been followed by anomalous or irregular symptoms. I can of course only hope to touch lightly upon a branch of such a vast subject, and any remarks I may make will relate only to those cases where an acute pneumonia has been followed by purulent effusion.

Is a lung solid with adventitious products or is the pleural cavity charged with fluid contents? This is a problem which we are often called upon to solve; happily it is one in which in the greater majority of cases we shall have, no doubt, no difficulty. On the other hand, we may be called upon to express an opinion where the clinical course has been shrouded in obscurity, where the symptoms from the first are complex and irregular, where our diagnosis is a matter not only of moment but of difficulty.

\footnotetext{
1 Abatrant of paper read before the Metropolitan Countits Branch, North London District.
}

The ambiguity which arises in those cases where the ordinary well-recognised symptoms of pleural effusion or solidified lung are either absent or profoundly modified have occupied the attention of many clinical observers. It is not easy to realise how completely a chest filled with fluid can simulate a lung solid from pneumonia. But a short time past we had a good instance of this in the case of a woman admitted into hospital suffering from cough and dyspnoea. Her right side was absolutely dull. On this side was the scar due to her breast having been removed for scirrhus. There were symptoms of recurrence of the growth. In this case the entire balance of physical evidence lent much weight to the opinion that the chest was filled with solid mass; an aspirator, however, quickly settled any doubt, and we found the cavity flled with fluid.

The difliculty in diagnosis is much increased if the primary seizure be of the nature of pneumonia, especially if the attack occur on the right side. A young girl, now in hospital, was admitted on February 7 th, 1888; she had been ailing about a week, had caught cold and had had a sharp shivering fit. I saw this patient on the afternoon of the day on which she was admitted her clinical condition was typical of an acute attack of pneumonia : respirations between 40 and 50; alæ nasi working vigorously; pulse 130 ; temperature $104^{\circ} \mathrm{F}$.

Beyond some sharp fine crepitation to be heard at the base of the left lung, the physical symptoms were insignificant. She had pain over this side, but no stitch or catch in breathing. The air appeared to be entering both sides of the chest equally well. The following day there was increase in the area over which fine crepitation could be heard, and the lower two-thirds of the left chest was dull ; no bronchial breathing could be detected; there was no expectoration, though frequent cough.

From this time the patient was severely ill, so ill that an accurate, careful examination of the chest was impossible. The lower part of the right lung was implicated in the attack, the constitutional symptoms being extreme.

On January 14th, seven days after admission, a thorough examination was made. The left side was dull, with tubular breathing and bronchophony. The apex-beat of the heart could not be felt. but the heart-sounds were heard loudest to the left of the median line. There had been no bectic, no shivering, the temperature gradually falling, and the general condition of the patient had improved. She pronounced herself as feeling much better. Nevertheless the breathing was still rapid and embarrassed. At this time the general condition of the patient suggested the presence of fluid within the chest, though a physical examination almost directly negatived such a view.

On January 17th the girl was seen again. She expressed herself as being much more comfortable, had slept well, and had taken food with appetite. The temperature was now normal, but both pulse and respiration were still rapid. Cough was very frequent, almost paroxysmal, but without expectoration. On this day I distinctly heard the heart-sounds loudest to the right of the median line. An aspirator was used, and a pint of sweet pus withdrawn. Subsequently a free opening into the chest was made by my colleague, Mr. Durham, about two pints of pus being removed. The patient has made an uninterrupted recovery.

Another case, bearing on the matter under our notice, occurred to me in the case of a patient admitted under my care into the West London Hospital, on March 5th, 1886, suffering from an attack of pneumonia of ordinary intensity. There was nothing irregular in the clinical course, and the normal physical symptoms of pneumonia were present. There was a rather free expectoration of rusty and blood-stained sputa.

On the seventh day after admission the general state of the patient was satisfactory. The attack had run an ordinary course, and on the morning of this day the temperature was 99\%. The percussion note at the lower part of the right chest was toneless, that is; it was more dull than is usually the case from pneumonic consolidation. The cough was irritating and paroxysmal. We had noticed the ordinary cough of pneumonia giving place to one which was paroxysmal and without expectoration. Such a cough may be caused by the presence of a small quantity of fluid within the chest. A hypodermic syringe revealed the presence of fluid. and a small quantity, namely four ounces, was removed, and proved to be sero-purulent. It was sweet. The following day the temperature was normal, the cough had gone, and the patient, a few days later, was discharged well.

But lately a child was under my care in the West London Hospital suffering from a slight attack of broncho-pneumonia. The 